\title{
DISPLACEMENT OSTEOTOMY OF THE UPPER END OF THE FEMUR
}

\author{
E. N. Wardle, LiverpoOl, ENglaNd
}

The present position of surgery of the hip has encouraged me to review the results in a series of patients for whom I performed a displacement osteotomy during the years 1932 to 1952. This record illustrates the useful application of the procedure to three major conditions-osteoarthritis of the hip, old ununited fracture of the neck of the femur, and old unreduced congenital dislocation or subluxation of the hip.

It is fair to say that the operation is derived from that originally described by Lorenz (1925), of Vienna, for adult patients with unreduced congenital dislocation of the hip. He called it a "bifurcation osteotomy." The name was apt. The long lower fragment of the femur was realigned in the normal weight-bearing axis and the short upper fragment was made to lie at an inclination parallel to the slope of the pelvic wall; the angle where the two fragments met pointed into the acetabulum. As a matter of interest, fourteen of the forty cases recorded here were cases of old unreduced congenital dislocations or subluxations, and, although the technique of Lorenz was not followed exactly, Figure 1 is typical of the result ten years after operation.

During the 19:0's patients with ununited fractures of the neck of the femur were treated in Liverpool by a similar osteotomy, but at the intertrochanteric level, with displacement inwards of the upper end of the shaft beneath the femoral head. Figure 2 illustrates a typical result fourteen years later.

McMurray (1939) described and used an oblique osteotomy with inward displacement of the femoral shaft for osteoarthritis of the hip. McFarland (1954) described the late results in a series of these patients. Twenty-one of my patients were operated upon for osteoarthritis; Figure 3 is a typical result eight years afterwards.

\section{GLINICAL MATERIAL}

Sixty-nine patients have been subjected to displacement osteotomy in the twenty years covered by this review. The average time since operation is eleven years. Forty of the sixty-nine patients have been examined during the last year; four are known to have died, and the remainder could not be traced. Of the forty patients that were examined twenty-one had osteoarthritis, fourteen congenital dislocation, and five ununited fracture of the neck of the femur.

\section{RESULTS}

The results have been assessed by the method described by Shepherd (19.54) and the grading of the forty patients examined is shown in Table I.

TABLE I

Results of Displacement Osteotomy in Forty Cases

\begin{tabular}{|c|c|c|c|}
\hline Excellent & Good & Fair & Poor \\
\hline 2 & 18 & 10 & 10 \\
\hline
\end{tabular}

The results according to the patient's reply when asked whether the operation had been worth while are shown in Table II. 


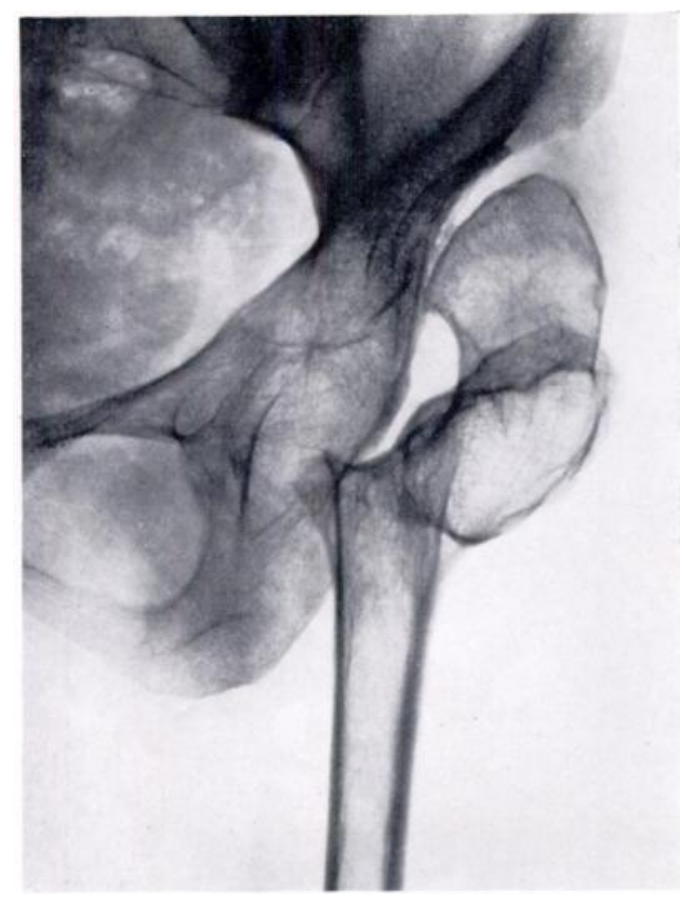

Fig. 1

Tipical late appearance of realignment osteotoms for unreduced congenital dislocation of the hip. The patient was aged forty-five at the time of operation. Her pain has been relieved.

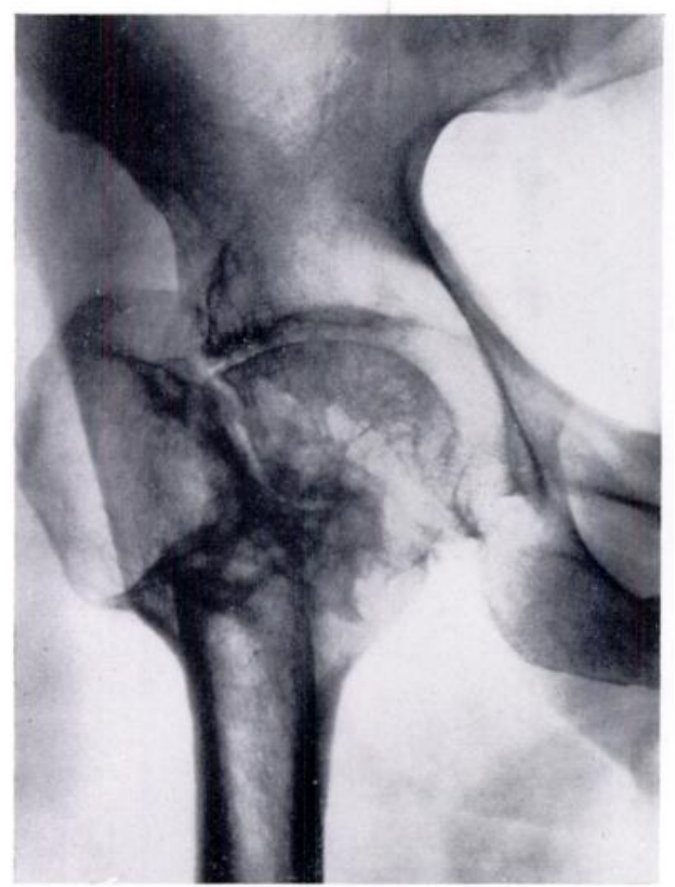

FIG. 2

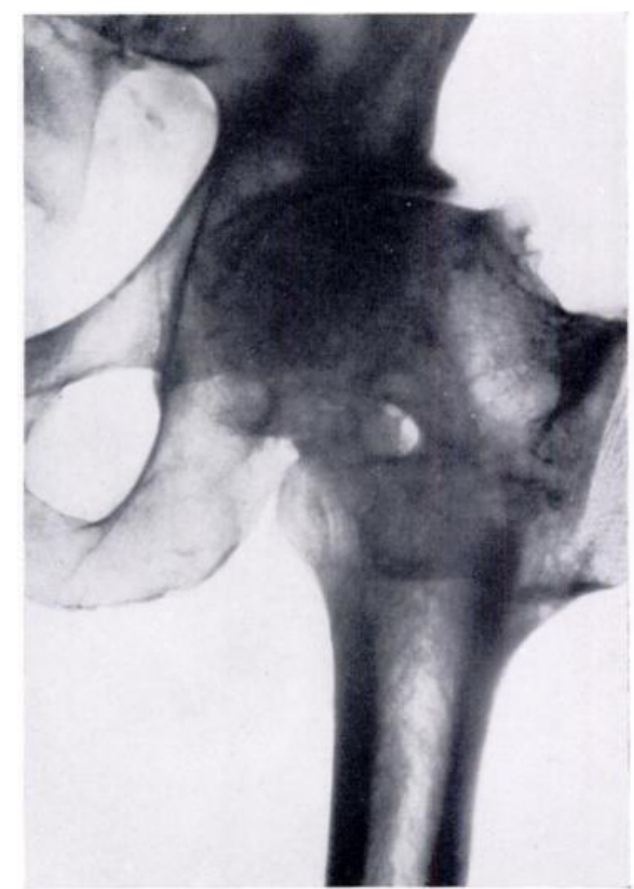

Fig. 3

Figure 2 - Late appearance of displacement osteotomy for ununited fracture of the neck of the femur. This patient has retained 90 degrees of flexion at the hip and walks without a stick. Figure 3 - Typical late appearance after displacement osteotomy for osteoarthritis. The relief of symptoms in the left hip enabled the patient to return to work in spite of the condition of his right hip.

VOL. $37 \mathrm{~B}$, NO. 4, NONENBER 1955 
A high proportion of patients in all three conditions are therefore satisfied with their results over a long period. This suggests that the operation should continue to be given serious consideration and that the factors making for success ought to be established. No claim is made that the osteotomy restores movement to a hip (although incidentally this

TABI.E II

Patients' Assessment of Reslet in Forty Cases

$\frac{\text { Enthusiastic "yes" }}{31} \frac{\text { Qualified "yes" }}{4}$ ". .o"

may occur) but it is claimed that the previous range is retained, that pain is removed or relieved, that deformity is corrected, and stability ensured. Even in an assessment that includes an index of mobility half the patients had good results - a record not yet reached after a similar lapse of time by any method of arthroplasty.

\section{DISCUSSION}

Probably the common factor for success whether in a patient with osteoarthritis, ununited fracture of the neck of the femur, or old congenital dislocation of the hip, is that the upper fragment was adducted and has remained so. This change in position of the head-and-

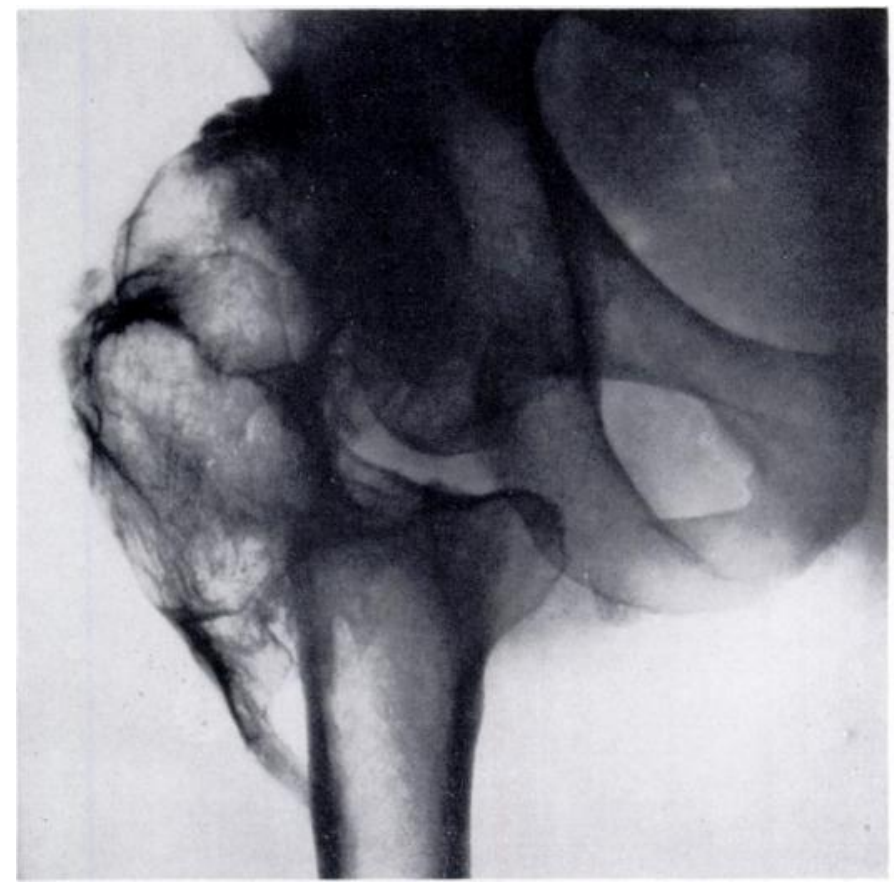

FIG. 4

To show the reversal of the normal varus angle into a valgus angle.

neck fragment was noted by Osborne and Fahrni (1950); but their emphasis was on the alteration of the area of weight bearing of the femoral head and not upon adduction of the upper fragment which I believe so essential. This is the position in which nature heals a hip joint. In order to take advantage of it, and at the same time restore the femoral shaft to weight-bearing line, the normal varus angle between the neck and shatl must be reversed 


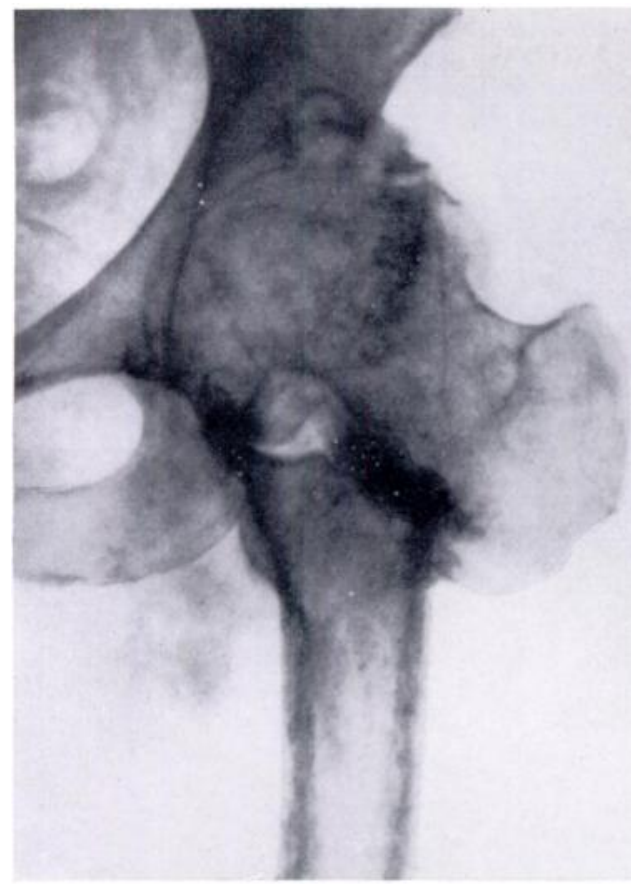

FIG. 5

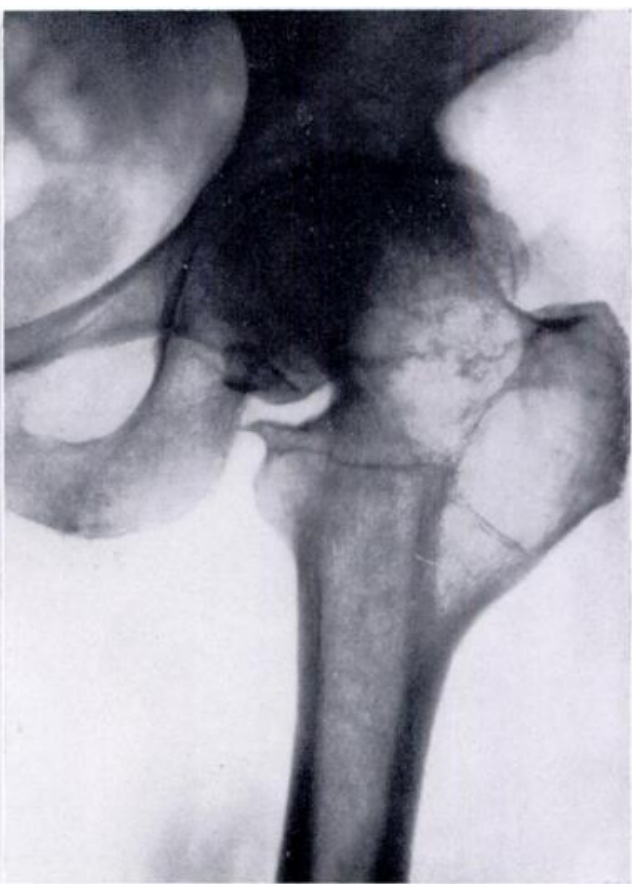

FIG. 6

(ase 1-l'atient aged fifty-eight. Figure 5-Three months after displacement osteotomy for osteoarthritis. Figure 6-Eight years later. Note increase of cartilage space.

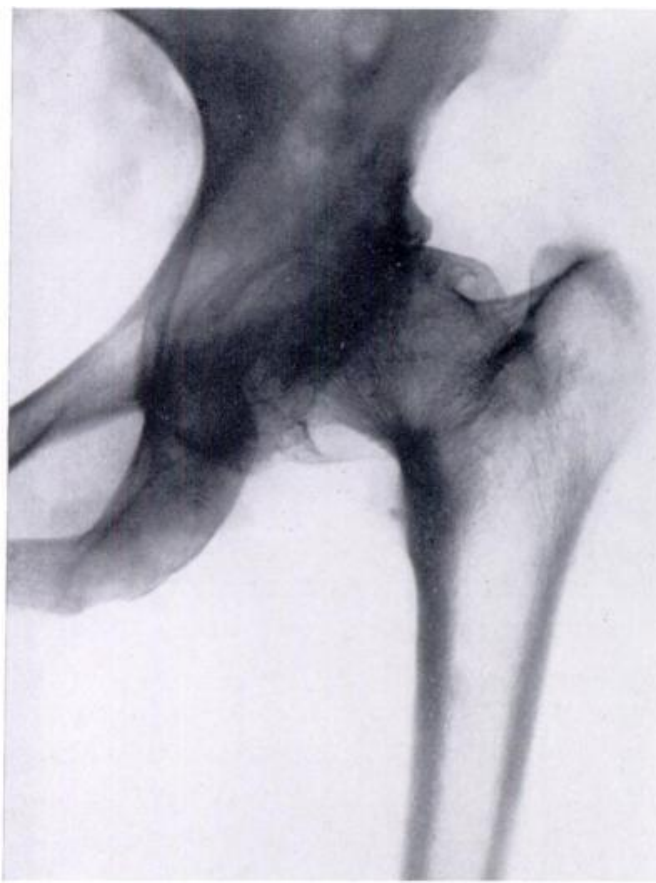

Fili. 7

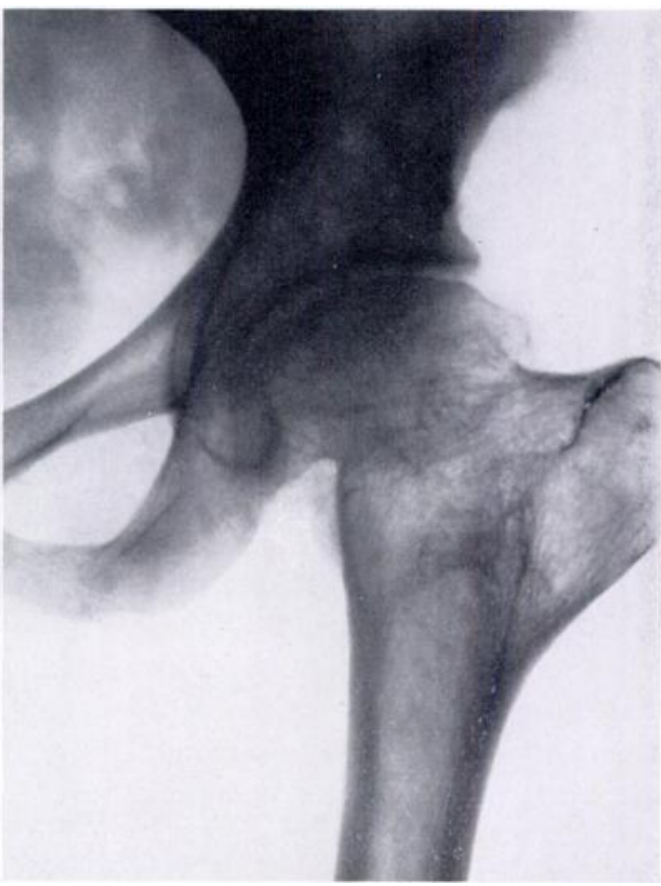

Fili. \&

Case 2 Patient aged sixty-two. Figure 7 Before operation. Figure 8 Four years after displacement osteotomy. Note reappearance of cartilage space.

V(1.. 37 B, No. 4 , NOVEMBER 1955 
into valgus (Fig. 4). Failure to produce operatively this precise relationship, with the upper fragment adducted and the lower fragment in weight-bearing line, impairs the result. I have termed the establishment of this desirable position " reciprocal realignment." If the correct realignment is achieved one notices in late radiographs a reappearance of the joint space previously obliterated in the arthritic patients (Figs. 5 to 8). This tendency of the bone and articular cartilage of the femoral head to return towards normal after a successful osteotomy suggests a profound metabolic change. This was referred to by McFarland (19:54). It is of interest also that in some cases of non-union of the femoral neck union of the fracture occurred after the osteotomy (Fig. 9).

Arguments adduced against the displacement osteotomy-The necessity to fix the hip in a plaster spica after operation is a disadvantage. No serious complications from this cause occurred in my series, but the patients were selected. The plaster spica prohibits the

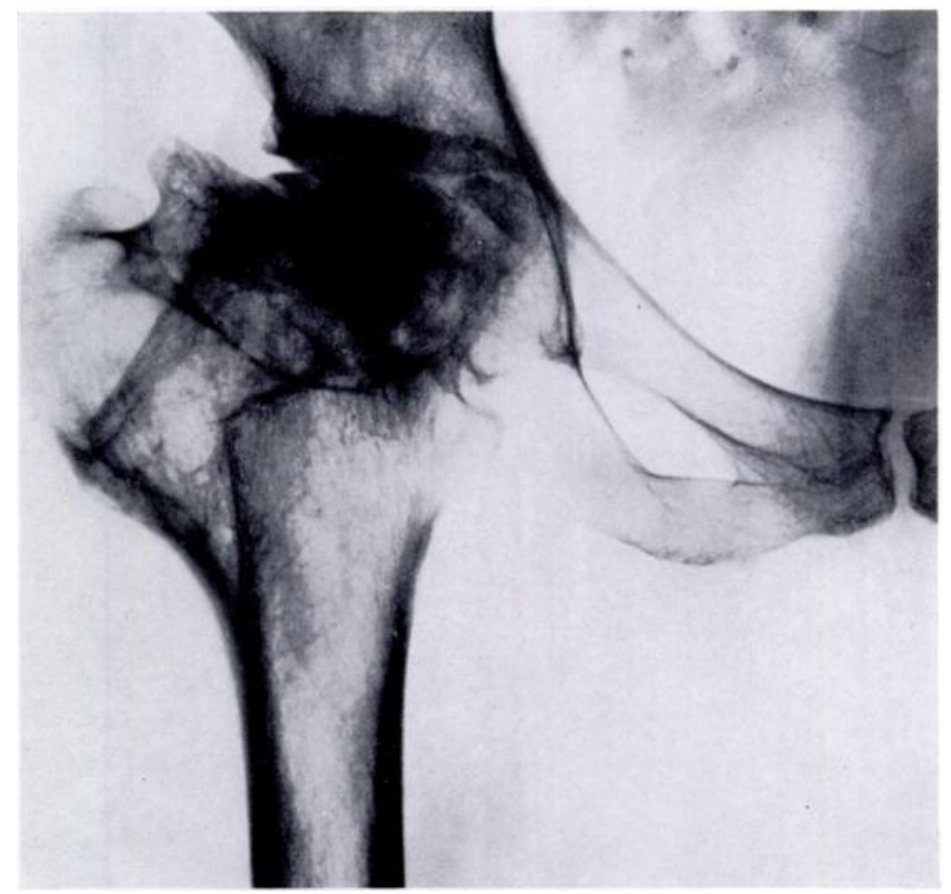

FIG. 9

Case 3-Result six years after osteotomy for ununited fracture of the neck of the femur.

operation in just those elderly patients for whom it is most appropriate. Disabling stiffness of the knee is often quoted as another adverse feature, but this is not supported by the present series of forty patients. The average range of movement of the knee on the affected side was 100 degrees. Eight patients had a full range of movement and twenty-five had 90 degrees. The average age of the patients was forty-nine years at the time of operation.

Mention has also been made of valgus deformity of the knee on the side of operation. None of my patients has been affected in this way; and, although it was observed to a slight degree, it was not of any functional significance and it is not an inevitable accompaniment of the displacement. It results from abducting the leg more than is necessary. The statement that because of this genu valgum the operation should not be performed on both hips in the same patient cannot be supported; the deformity will occur only if the femoral shafts are over-abducted and immobilised for the whole period in that position. More importance should be attached to performing the osteotomy correctly. Experience leads me to believe 
that most of the poor results are due to guessing the angle and site of the osteotomy and to the splintering that may occur at the inner cortex if it is divided blindly.

Technique of operation--Originally I used a straight incision beginning at the greater trochanter and extending five inches down the outer side of the thigh. The upper part of the shaft of the femur was exposed and divided just below the greater trochanter, the line of osteotomy running upwards and medially towards the lesser trochanter. With the osteotome still in position, the limb was abducted, the osteotome being used like a shoe-horn to displace inwards the upper end of the lower fragment. After closure of the wound a plaster spica was applied with the limb abducted to 45 degrees and the knee slightly flexed; the plaster included the foot. As a rule union was sufficiently advanced in two months for the patient to walk, though commonly a short plaster spica was applied for a further month or so.

I gradually modified this procedure in order deliberately to adduct the upper fragment, because, as I have indicated, a diseased hip left to natural processes tends to settle into

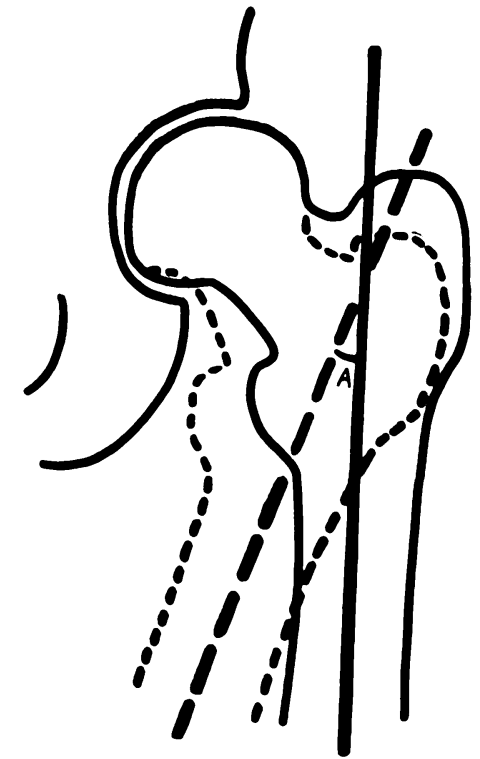

Fig. 10

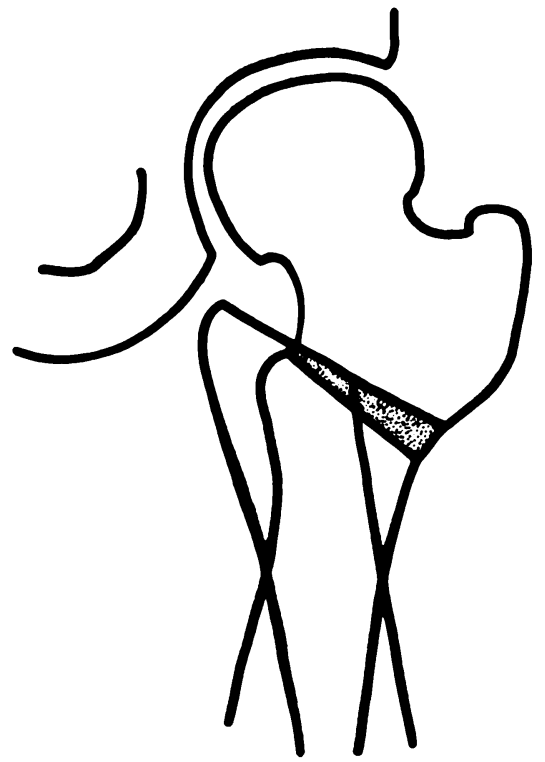

Fig. 11

ligure 10- The angle $A$ is measured before operation and the lower fragment is abducted through an equal angle at the site of osteotomy. Figure 11 - If the hip has no free range of adduction the lower fragment is angled and displaced medially as shown.

adduction as it heals. Before operation the leg is adducted as much as possible and the angle that the shaft of the femur makes with the anatomical position is noted (A in Figure 10). This indicates the angle through which the shaft must be moved in the direction of abduction to take up the anatomical position against an upper fragment in full adduction. Since the upper fragment does not usually lie in the adducted position during the operation, after the osteotomy the shaft is abducted through angle $\mathrm{A}$ upon an upper fragment in neutral position. The hip is immobilised in this position. When union has occurred, and the leg is brought into anatomical or weight-bearing position, the upper fragment will be adducted and the reversal of varus into valgus attained (Fig. 4). If, before operation, the hip is already fixed in adduction it is only necessary to fix the limb, that is the lower fragment, in the neutral position after osteotomy:

If the hip has no free adduction movement or is fixed without deformity, then displacement and abduction must of themselves create the apex pointing to the acetabulum, and it may be necessary to remove some bone at the site of osteotomy to obtain this position

VOL. $37 \mathrm{~B}$, NO. 4, NOVEMBER 1955 
(Fig. 11). Figure 12 illustrates that when full adduction of the upper fragment is present or possible the line of osteotomy will be at right angles to the long axis of the femoral shaft. When no adduction is present the line of osteotomy becomes oblique (Fig. 13). These are extreme examples.

It is important to expose enough of the femur to be able to choose exactly the site of osteotomy. This varies with the lesion in question and should be so chosen that after displacement the apex of the new angle between neck and shaft will point at the lower border of the acetabulum. The site of osteotomy is therefore lower down the shaft in congenital dislocation, nearer the lower border of the greater trochanter in ununited fracture of the neck of the femur, and practically through the lower border of the trochanter in osteoarthritis.

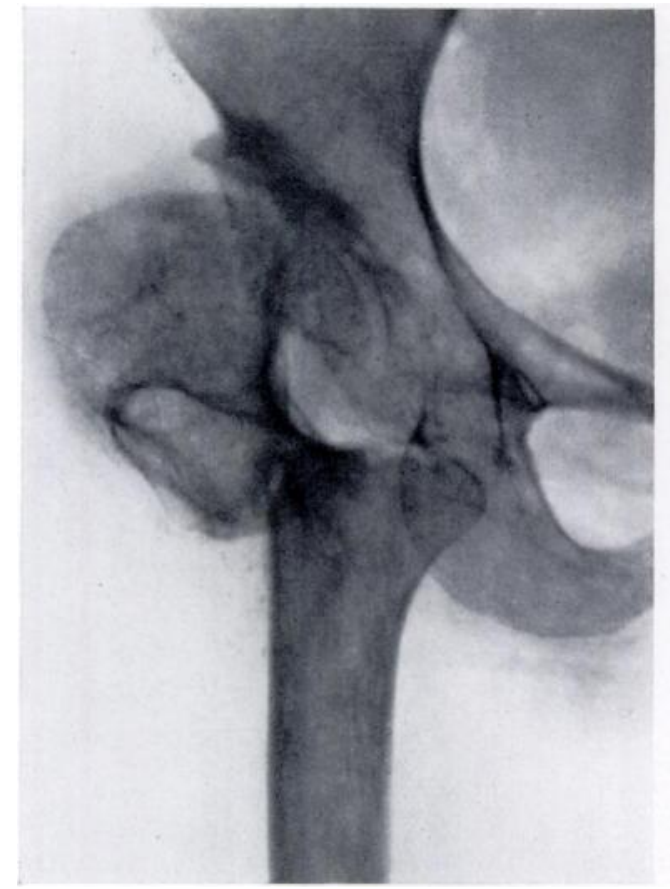

FIG. 12

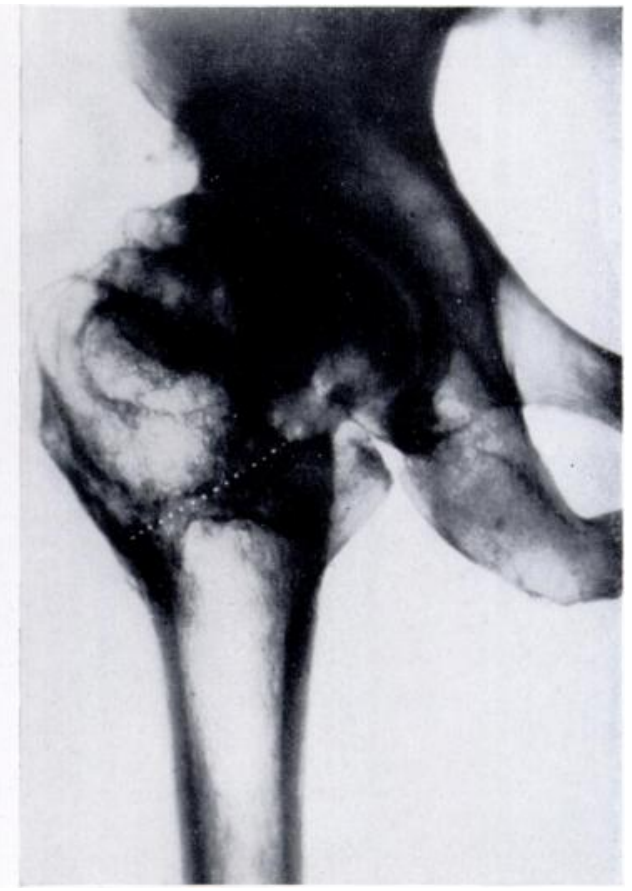

FIG. 13

Figure 12 - When the upper fragment lies fully adducted the line of osteotoms is at right angles to the axis of the femoral shaft. Figure 13 - When adduction of the upper fragment is absent the line of osteotomy is oblique.

The medial end of the line of osteotomy will be just below, through, or just above the lesser trochanter according to the obliquity required.

To ensure the desired adduction of the upper fragment, the correct degree of alduction of the lower fragment in relation to the upper fragment, and the precise selection of site and angle of osteotomy, the exposure was modified to attain a clear view of the anterior and lateral surfaces of the femur for one inch above and one inch below the intertrochanteric line, and to expose and make accessible the inner border of the femur above and below the lesser trochanter. The skin incision therefore extends from a point just below and lateral to the anterior superior spine downwards and backwards to the greater trochanter, and then turns down the middle of the outer side of the thigh for about six inches. The muscles attached to the anterior and lateral aspects of the trochanter and of the adjacent shaft of the femur are reflected from the bone sufficiently to give the exposure described above. The line of osteotomy is then marked as a groove across the front of the femur, and at its inner end a small osteotome is driven through the calcar femorale antero-posteriorly in the transverse plane. Tlis 
precludes the persistence of a spike of bone attaching to the lower fragment which can block displacement (Fig. 14). A broad osteotome is then driven from the lateral surface through the femur along the predetermined line. The leg is abducted and the osteotome is used as a lever to obtain displacement in the manner already described.

In order to avoid plaster immobilisation, and so make the operation suitable to those elderly patients for whom it is so clearly indicated, internal fixation has been increasingly

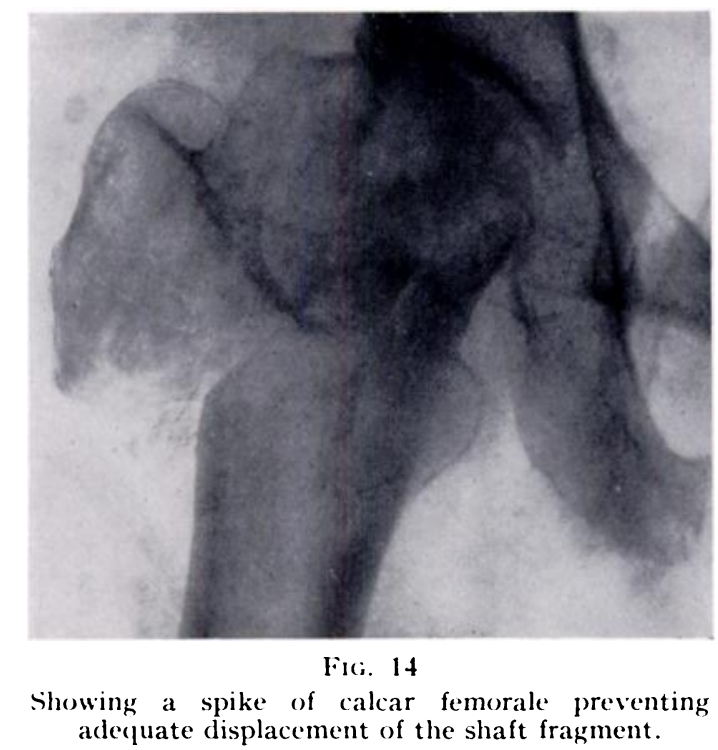

used during the last three years. A nail-plate has been used which can be accommodated to the various angles required and yet is rigid enough to allow the patient to be nursed in bed without any external fixation.

\section{SUMMARY}

1. Forty patients out of sixty-nine operated upon between 193:2 and 19:52 by displacement osteotomy for congenital dislocation of the hip, ununited fracture of the neck of the femur, and osteoarthritis of the hip have been reviewed and the results analysed.

2 . The factors responsible for the success of the operation are discussed and the technique of operation is described.

My thanks are offered to Professor Bryan McFarland for his helpful and constructive criticism, and to my registrars Messrs ( $i$. A. Wetherell and iv. A. I. Thompson for interviewing and assessing my patients.

\section{REFERENCES}

LORENZ (1925): Verhandlungen der Deutschen Orthopädischen (iesellschaft. 19 kongress, 129. Stuttgart: Ferdinand Enke.

McFartaxi), B. (1954): My Present Attitude to ()steo-arthritis of the Hip. Journal of Bone and Joint Surgery, 36 A, 476.

McllurkaY, T. P. (1939): Osteo-arthritis of the Hip Joint. Journal of Bone and Joint Surgery, $21,1$.

OSborne, (. V., and Fahrni, W. H. (1950): Oblique Displacement Osteotomy for Osteoarthritis of the Hip Joint. Journal of Bone and Joint Surgery, 32 B, 148.

SHEPHERD, M. M. (1954): Assessment of Function after Arthroplasty of the Hip. Journal of Bone and Joint Surgery, 36 B, 354.

VOL. 37 B, NO. 4, NOVEMBER 1955 